\title{
Social domain based modulation of neural responses to threat: the different roles of romantic partners versus friends
}

Article

Accepted Version

Morriss, J., Bell, T., Johnstone, T., Van Reekum, C. M. and Hill, J. (2019) Social domain based modulation of neural responses to threat: the different roles of romantic partners versus friends. Social neuroscience, 14 (4). pp. 398-408. ISSN 1747-0927 doi:

https://doi.org/10.1080/17470919.2018.1486735 Available at https://centaur.reading.ac.uk/77803/

It is advisable to refer to the publisher's version if you intend to cite from the work. See Guidance on citing.

To link to this article DOI: http://dx.doi.org/10.1080/17470919.2018.1486735

Publisher: Taylor \& Francis

All outputs in CentAUR are protected by Intellectual Property Rights law, including copyright law. Copyright and IPR is retained by the creators or other copyright holders. Terms and conditions for use of this material are defined in the End User Agreement. 


\section{CentAUR}

Central Archive at the University of Reading

Reading's research outputs online 
Running Title: Social domains

\section{Social domain based modulation of neural responses to threat: The different} roles of romantic partners versus friends

Jayne Morriss ${ }^{1}$, Tiffany Bell ${ }^{1}$, Tom Johnstone ${ }^{1}$, Carien M. van Reekum ${ }^{1}$, and Jonathan Hill ${ }^{*}$

${ }^{1}$ Centre for Integrative Neuroscience and Neurodynamics

School of Psychology and Clinical Language Sciences

University of Reading

Reading

UK

${ }^{2}$ School of Psychology and Clinical Language Sciences

University of Reading

Reading

UK

* Corresponding author:

Email: j.e.morriss@reading.ac.uk

Word count: 5,650 


\begin{abstract}
The neural circuitry associated with threat regulation in the absence of other people is well established. An examination of threat regulatory processes with people from different domains of an individual's social world is key to understanding social emotion regulation and personality functioning conceptualised as social domain organisation. In this study, 42 healthy female participants completed functional magnetic imaging sessions in which they underwent a scan in the presence of a romantic partner or friend, whilst completing a threat of shock task. In the presence of a romantic partner vs. friend, we found a reduction in amygdala activation to threat vs. safe trials over time. Furthermore, in the presence of a romantic partner vs. friend we observed greater subgenual anterior cingulate cortex and ventromedial prefrontal cortex activation to threat vs safe trials overall. The results support the hypothesis that recruitment of threat regulation circuitry is modulated by romantic partner relative to another person well-known to the individual. Future work needs to examine neural responses to a wider range of stimuli across more social domains, and implications of failures of this neural organisation for psychopathology.
\end{abstract}

Keywords: Social domains, romantic relationship, friendship, threat regulation, amygdala, prefrontal cortex 
Running Title: Social domains

\section{Introduction}

According to the social domains hypothesis, we classify others' behaviours and communications using a limited number of categories that we refer to as domains, in order to speed information processing and underpin rapid social action and interaction (Hill, Pilkonis, \& Bear, 2010). This domains based organisation is found in interactions with the same people within families (Hill, Fonagy, Safier, \& Sargent, 2003; Hill et al., 2014) and with different people for example partners, friends, work colleagues and others met in wider social interactions (Hill et al., 2008). We have also argued that this is not only a perceptual classification, but also one that entails an organisation of emotion regulatory strategies in anticipation of the likely regulatory resources available in the domain.

The social domains hypothesis is embedded within the broader conceptual view that responses to stimuli throughout biological systems entail an interplay between representation of their salient features and action (Bolton, 2004). In humans, "social" heuristics involve both social attributional and emotion regulatory processes and they are seen early in development. For example from around the age of 9 months distressed infants are likely to seek comfort from a parent, but to become more distressed in the presence of a stranger (Sroufe, 1977). They are already using a social domains heuristic. How this social domains heuristic is acquired is not known. However most children growing up in a supportive environment show a social domains based differentiation of emotional and behavioural responses, and by contrast many children who experience early serious privation in institutional care show deficits in a domains based organisation of behaviours towards adults (Kennedy et al., 2017). Acquiring the ability to respond to 
Running Title: Social domains

social contexts according to their domain may therefore be central to personality development (Hill, Pilkonis \& Bear 2010). This is consistent with personality theories that seek to account both for stable individual differences and situational variability, by proposing predictable patterns of variability across situations, mediated via cognitive-affective processes (Mischel \& Shoda, 1995). The deployment of different cognitive-affective processes provides the match between those processes and the demands or opportunities provided by the situation. Consistent with our hypothesis that key situational variations are defined by social domains we found that secure attachment in adults, which requires evidence of effective regulation of emotions in close relationships, is associated with successful romantic, but not with friendship functioning, in both general population and clinical samples (Hill et al., 2011). Failures to maintain the behavioural and emotion regulatory demarcation across domains, i.e. 'domain disorganisation', are more common in adults with borderline personality disorder than in other psychiatric patients (Hill et al 2008, Morse et al 2009). Thus if social domains identify a social information heuristic used to inform emotion regulatory strategies, then the mere presence of another person will be sufficient to demonstrate a domains based modulation of neural activations indicative of those strategies.

Whilst not examining it from a domains based perspective, previous research using brain imaging has focused on how threat regulation may be modulated differently depending on the presence of someone else (Coan et al., 2017; Coan, Kasle, Jackson, Schaefer, \& Davidson, 2013; Coan, Schaefer, \& Davidson, 2006; Eisenberger et al., 2011; Kawamichi, Kitada, Yoshihara, Takahashi, \& Sadato, 2015; Maresh, Beckes, \& Coan, 2013; Younger, Aron, Parke, Chatterjee, \& Mackey, 2010). 
Running Title: Social domains

Typically, when alone, threat regulation has been found to involve the coordinated action of specific brain regions including the amygdala and parts of the subgenual anterior cingulate and ventromedial prefrontal cortex (Etkin, Egner, \& Kalisch, 2011; Sehlmeyer et al., 2009). Generally, the amygdala is thought to be responsible for signalling threat (and more broadly salience) and parts of the ventromedial prefrontal cortex are thought to be responsible for signalling safety. Over the course of time, amygdala activation is susceptible to threat habituation (Büchel, Morris, Dolan, \& Friston, 1998; Fischer et al., 2003; LaBar, Gatenby, Gore, LeDoux, \& Phelps, 1998). Several studies have also shown differences in neural activity during the anticipation of threat, when holding the hand of a romantic partner versus a stranger, such as suppressed activity in the insula and dorsal anterior cingulate, areas associated with threat and pain (Coan, Schaefer, \& Davidson, 2006; Coan et al. 2017). In addition, these studies have also identified suppressed activity in inferior and lateral parts of the prefrontal cortex, areas associated with inhibition and attention, when holding the hand of a romantic partner versus a stranger. In studies that provided visual cues of the romantic partner rather than physical contact, increased activation to painful stimuli in areas thought to support safety or reward signalling, including the ventromedial prefrontal cortex, has been observed, relative to various visual control cues (stranger, acquaintance, object) (Eisenberger et al., 2011; Younger et al., 2010). Moreover, neural activation patterns during threat of shock tasks are further influenced by relationship quality and mutuality, such that better relationship quality and mutuality between romantic partners was associated with reduced activation in threat and pain-related regions when holding the hand of romantic partners (Coan et al., 2006), and increased activation in safety signalling regions when viewing pictures 
Running Title: Social domains

of romantic partners (Eisenberger et al., 2011). Recent studies have also shown modulation of neural responses to threat when holding the hand of a friend (Kawamichi, Kitada, Yoshihara, Takahashi, \& Sadato, 2015; Maresh, Beckes, \& Coan, 2013). In these studies, holding the hand of friend compared to rubber hand or the hand of a stranger has been shown to suppress visual cortex activity to negative stimuli (Kawamich, Kitada, Yoshihara, Takahashi, \& Sadato, 2015), as well as enhance activity in threat-related brain regions associated with engagement in anxious individuals (Maresh, Beckes, \& Coan, 2013). Taken together these studies provide evidence that neural activations are modulated by social processes and this modulation may vary depending on the identity of the other person, the experimental procedure to capture social presence, and the type of "threat".

Building upon these prior findings and using the social domains perspective we expect neural activations associated with presence of the other during scanning whilst participants anticipate shock to reflect a domains-based organisation of threat regulation. This, we hypothesise, occurs prior to 'load sharing' with another which might be captured by physical contact procedures such as hand-holding. Neural activations to threat contrasting the presence of an affiliate from two different social domains, without physical contact, would extend prior findings by highlighting the role that different social partners can play in supporting regulation of threat.

The aim of the current study was to investigate the neural mechanisms underpinning threat regulation in the presence of others from the social domains of romantic relationship and friendship. We recorded functional MRI during an instructed threat of shock task, and obtained subjective ratings of participants' experiences of the stimuli. Patterns of brain activation during the presence of a 
Running Title: Social domains

romantic partner versus friend were then compared for threat of shock versus safety from shock overall, and across time to capture effects of habituation. Based on the domains hypothesis that attachment processes are seen in romantic partnerships but not in friendships, we expected evidence of increased engagement of a threat regulation network (i.e. reduced amygdala activity and increased medial prefrontal cortex activity) in the presence of a romantic partner compared to a friend, particularly in the early trials compared to late trials.

\section{Method}

\section{Participants}

Participants included those who were scanned and "affiliate" participants (romantic partners or friends). Participants that were scanned consisted of 42 female volunteers recruited from the University of Reading and local area through advertisements $(\mathrm{M}$ age $=33 \mathrm{yrs}, \mathrm{SD}$ age $=7.33 \mathrm{yrs})$. We restricted the sample of participants to females in this first study, to reduce any possible sex-related variance in fMRI, and in line with prior work (Coan et al., 2006; Eisenberger et al., 2013). To be eligible for the study participants had to be free of contraindications for MRI, be right-handed, have normal or corrected to normal vision, report no current psychiatric illness, and be medication-free. In order to ensure that any differences in emotion regulation by partner or friend was currently relevant to participants, they had to have a romantic partner and a friend of at least 3 years standing, both of whom were available to attend for scanning as affiliate participants. Requiring female participants only to bring male friends would have removed a sex-difference confound, but would 
Running Title: Social domains

have reduced generalisability in two ways. First, the majority of friendships are same-sex, and second, opposite sex friendships often entail sexual attraction and romantic interests (Bleske-Rechek et al., 2012). We therefore allowed participants to bring either a same sex or opposite sex friend. Whether the affiliate participant was a partner or friend was allocated at random, with the aim of generating two groups of participants with similar relationship networks. Affiliate participants consisted of 42 volunteers $(M$ age $=34.26$ yrs, $S D=7.62$ yrs $) .22$ participants brought were the romantic partner of a corresponding participant $(M$ time known $=10.23$ yrs; $S D=6.04$ yrs, 22 males) and 20 participants brought were the friend of a participant ( $M$ time known $=8.13 \mathrm{yrs} ; S D=5.80 \mathrm{yrs}, 3$ males, 17 females). 41 participants had an opposite sex romantic partnership, 1 participant had a same sex romantic partnership (who was randomly allocated to the "friend" condition). The age of the participants and the length of time the participants had known the affiliate participants did not significantly differ between the romantic partner or friend groups, $t(40)=.299 p=.76 ; t(40)=1.074 p=.28$.

All participants provided written informed consent and received $£ 15$ for their participation. The University of Reading's Research Ethics Committee approved the study protocol.

\section{Procedure}

On arrival, the participants were separated. The participant was taken to the scanning unit, whilst the affiliate was taken to another testing location within the same building. Both participants were given: (1) consent forms to read and sign; (2) information sheets that outlined their roles in the experiment; (3) questionnaires to 
Running Title: Social domains

complete; (4) a 5 minute interview on their relationship with the other participant; (5) a practise of the threat of shock and picture tasks on a computer. The practise included letters and pictures that were representative of the ones shown in the tasks.

Next, participants were taken to the MRI scanner where they underwent scanning while performing a threat of shock task (and a picture viewing task part of a separately reported experiment). Participants were instructed to maintain attention to the tasks and to keep as still as possible. In the first half of the experiment, the participants completed both tasks as part of a separately reported experiment on emotion regulation and individual differences. In the second half of the experiment, the participant completed the tasks whilst their partner or friend was present in the scanning unit. Before each task began, the participant talked to their partner or friend for 2 minutes via webcam about the tasks they had completed before their affiliate joined them, and how they had found them. At the end of participants' conversations we reminded the participant that their partner or friend was in scanner control room. The participant was aware that their partner/friend could see them and the screen that they would be viewing. After scanning, the participants rated the threat of shock stimuli (and picture stimuli - data not reported here) outside of the scanner.

\section{Threat of shock task}

The task was administered using E-Prime 2.0 (Psychology Software Tools Ltd, Pittsburgh, PA). At the start of the threat of shock task with the affiliate present, the participant and affiliate could communicate across a webcam and intercom for 2 minutes. 
Running Title: Social domains

Visual stimuli were presented using a mirror on the head coil positioned towards a BOLDscreen LCD monitor (Cambridge Research Systems). Screen resolution was set at $800 \times 600$ pixels.

The possibility of receiving an electric shock to the index and middle finger of the dominant hand was used to induce anxiety. Electric shocks were delivered via ADInstruments PowerLab 26T Isolated Stimulator using MRI-safe MLT117F Ag/AgCl bipolar finger electrodes. Each participant's stimulation level was set by first exposing them to an electric stimulation of $1 \mathrm{~mA}(10$ pulses at $50 \mathrm{~Hz}$, with a pulse duration of $200 \mu \mathrm{s}$ ) and increasing the current in steps of $0.5 \mathrm{~mA}$, up to a maximum of $10 \mathrm{~mA}$. This continued until a suitable participant-specific threshold was found that was uncomfortable but not painful. This level was then used throughout the threat of shock task for that subject (electric stimulation level: $M=2.21 \mathrm{~mA} ; S D=1 \mathrm{~mA}$ ). The level of shock used was similar across partner $(M=2.27 \mathrm{~mA} ; S D=1.08 \mathrm{~mA})$ and friend $(M=2.15 \mathrm{~mA} ; S D=0.91 \mathrm{~mA})$ groups and did not significantly differ, $t(40)=.366$ $p=.71$.

Participants were required to view cues that represent either threat of shock or safety from shock. Only two cues were presented, a threat cue where there was $50 \%$ chance of receiving a shock and a safety cue where there was $0 \%$ chance of receiving a shock. Participants were informed which cues would signal threat of shock or safety from shock at the beginning of the experiment. Each trial consisted of: a white cue (e.g. X, O, D, T) presented on a black background (1 second), a white fixation anticipation cue presented on a black background ( 8 seconds), a small circle cue signalling the end of the trial ( 1 second) and a black blank screen (4-6 seconds) (see Figure 1). Participants completed 1 run of 36 trials (18 Threat, 18 Safe) with a 
Running Title: Social domains

partner or friend present (after a run of 36 trials alone, as reported under Procedure). The stimuli were counterbalanced across partner/friend runs. All experimental trials within the task were randomized.

\section{Questionnaires}

Levels of anxiety in the two groups were compared using the State-Trait Anxiety Inventory, Trait Version (STAIX-2) (Spielberger, Gorsuch, Lushene, Vagg, \& Jacobs, 1983). There was no significant difference in trait anxiety between those who attended with a partner $(M=40.05 ; S D=10.01)$ and those who attended with a friend $(M=41.90 ; S D=10.80 \mathrm{~mA}), t(40)=-.577 p=.56$.

The hypothesised attachment based difference between partner and friend relationships was assessed in two ways by self-report. In the first participants and affiliates were administered two 11 point Likert scales to assess attachment processes indicating how often they offered comfort to each other when distressed, and how often they confided in each other: 'We look to one another for comfort when we are worried or upset' and 'We confide in each other'. In the second they reported on how they would behave in contrasting domains. This was assessed using the Domain Emotion Expression Profile (DEEP) also administered to participants and affiliates, which comprises a one-page matrix of items covering 5 domains and 5 emotional and behavioural foci asking how they think they would behave in each of the 25 combinations (Jones, 2008). We addressed participant anticipations of attachment relevant behaviours by contrasting their scores on the item 'I would show upset (e.g. crying) and look for comfort' in relation to partners and friends. 
Running Title: Social domains

\section{Ratings}

Upon completion of the tasks in the scanner, main participants rated the valence and arousal of the cues that signalled threat of shock or safety from shock using a 9 point Likert scale ranging from 1 (Valence: very negative; Arousal: calm) to 9 (Valence: very positive; Arousal: excited).

Ratings data from the threat of shock task were reduced for each participant by calculating their average responses for each experimental condition. Missing data points were excluded.

\section{Ratings analysis}

Ratings of comfort seeking and confiding were compared using independent samples t-tests for each item by Group (participants and their affiliate partners vs participants and their affiliate friends). The rating from the DEEP related to demarcation between comfort-seeking in partner and friend relationships was examined using a Domain (comfort-seeking from partner, comfort-seeking from friend) x Group (participants and their affiliate partners vs participants and their affiliate friends) repeated measures ANOVA.

For the threat of shock task, we assessed contingency and social relationship effects by conducting a Contingency (Threat, Safe) x Presence (Partner, Friend) ANOVA for behavioral ratings.

\section{MRI}

Participants were scanned with a 3T Siemens Trio set up with a 12 channel head coil (Siemens Inc., Erlangen, Germany). T2*-weighted echo planar imaging 
Running Title: Social domains

(EPI) functional scans were acquired for each run of the threat of shock task consisting of 281 volumes respectively $\left(T R=2000 \mathrm{~ms}, \mathrm{TE}=30 \mathrm{~ms}\right.$, flip angle $=90^{\circ}$, FOV $=192 \times 192 \mathrm{~mm}, 3 \times 3 \mathrm{~mm}$ voxels, slice thickness $3 \mathrm{~mm}$ with an interslice gap of $1 \mathrm{~mm}, 30$ axial slices, interleaved acquisition). For more information on coverage of scanning sequences see Supplementary material.

Following completion of the functional scans, fieldmap and structural scans were acquired, which comprised of a high-resolution T1-weighted anatomical scan $\left(\mathrm{MP}-\mathrm{RAGE}, \mathrm{TR}=2020 \mathrm{~ms}, \mathrm{TE}=2.52 \mathrm{~ms}\right.$, flip angle $=90^{\circ}, \mathrm{FOV}=256 \times 256 \mathrm{~mm}, 1$ x $1 \times 1 \mathrm{~mm}$ voxels, slice thickness $1 \mathrm{~mm}$, sagittal slices), and two gradient echo fieldmaps $\left(\mathrm{TR}=488 \mathrm{~ms}, \mathrm{TE} 1=4.98 \mathrm{~ms}, \mathrm{TE} 2=7.38 \mathrm{~ms}\right.$, flip angle $=60^{\circ}, \mathrm{FOV}=$ $256 \times 256 \mathrm{~mm}$, slice thickness $4 \mathrm{~mm}$ with an interslice gap of $4 \mathrm{~mm}, 30$ axial slices)

\section{FMRI analysis}

FMRI analyses were carried out in Feat version 5.98 as part of FSL (FMRIB's Software Library, www.fmrib.ox.ac.uk/fsl). Brains were extracted from their respective T1 images by using the FSL Brain Extraction Tool (BET) (Smith, 2002). Distortion, slice timing and motion correction were applied to all extracted EPI volumes using FUGUE and MCFLIRT tools. Gaussian smoothing (FWHM 5mm) and a 100 second high pass temporal filter were applied.

Separate first-level GLM analysis was carried out for each functional scan run in the presence of a partner or friend from the threat of shock task. Separate regressors were specified for the experimental conditions of primary interest by convolving a binary boxcar function with an ideal haemodynamic response (HR), which corresponded to the length of trial (9 seconds). We examined the overall effect 
Running Title: Social domains

of threat of shock (Threat, Safe), as well as the temporal effects of threat of shock (Early Threat, Late Threat, Early Safe, Late Safe). Examination across time is a common analysis used to assess threat extinction and habituation (Büchel et al., 1998; Fischer et al., 2003; LaBar et al., 1998). We defined early as the first nine trials and late as the last nine trials for each condition. Regressors for the six motion parameters were included to model out variance due to uncorrected motion.

We defined two main effect contrasts to reveal anticipation to threat of shock. We examined the overall effect: Threat of shock: (Threat $>$ Safe), (Safe $>$ Threat). To examine threat of shock over time, we contrasted the first half of the run versus the second half of the run: Threat of shock: (Threat $>$ Safe)EARLY> (Threat $>$ Safe) LATE. All contrasts were normalized and registered to MNI standard space using FLIRT (Jenkinson, Bannister, Brady, \& Smith, 2002).

Second-level GLM analysis consisted of regressors for the whole group and the presence of a Partner or Friend using FSL's Randomise with Threshold Free Cluster Enhancement estimated from 5000 permutation samples and corrected at $p$ $<0.05$.

Based on predictions that there will be differences in threat related responding according to who is present during scanning, we used anatomically defined masks from the Harvard-Oxford cortical and subcortical structural atlases in FSL to select the left amygdala, right amygdala, subgenual anterior cingulate cortex and ventromedial prefrontal cortex regions with a $50 \%$ probability threshold. Small volume corrections on these regions were performed using FSL's Randomise with Threshold Free Cluster Enhancement estimated from 5000 permutation samples and corrected at $p<0.05$ based on the number of voxels within each mask. 


\section{Results}

\section{Self-report ratings}

Participants and their partners reported higher mean rates of comfort giving and confiding, than partiipants and their friends, t's $>5.3, p$ 's $<.001$ (see Table 1). All participants and affiliates reported that they would be more likely to show distress and seek comfort with a partner than with a friend, $F(1,82)=92.248, \mathrm{MSE}=28.916$, $p<.001$ (see Table 1). This demarcation in showing distress and seeking comfort with a partner over a friend, did not significantly differ depending on what group participants were allocated to, $F(1,82)=.313$ MSE $=.414, p=.521$.

Participants reported significantly greater negativity and arousal during threat vs. safe cues, $F(1,39)=16.172, \mathrm{MSE}=47.726, p<.001 ; F(1,39)=38.205, \mathrm{MSE}=$ 123.206, $p<.001$. There were no significant differences in the valence or arousal ratings in the presence of a partner or friend, $F(1,39)=2.364$, MSE $=6.750, p=$ .132; $F(1,39)=2.235, \mathrm{MSE}=7.206, p=.143($ see Table 2$)$.

\section{fMRI}

Main effects of threat of shock: When with another person, threat vs. safe cues induced greater activation in the bilateral insula, frontal operculum, and bilateral frontal orbital cortex, (see Table 3). The reverse contrast, safe vs. threat cues, revealed greater activation in the occipital cortex, precuneus and hippocampus (for full list of brain regions see Table 3). 
Running Title: Social domains

Social domain effects of threat of shock: We found significantly greater activity in areas of the subgenual anterior cingulate cortex and ventromedial prefrontal cortex to threat vs safe cues for the partner group compared to the friend group, without an effect of time (early vs late; see Table 3 \& Figure 2). Participants in the presence of a partner, compared to a friend, showed significant differences between early and late trials in threat vs safe activation (i.e. a group $x$ threat $x$ time interaction) in the left and right amygdala (see Table 3 \& Figure 3). We then extracted contrast estimates from the significant clusters and performed follow-up pairwise comparisons to identify the source of the (Threat $>$ Safe) EARLY $>$ (Threat $>$ Safe) LATE X Group interaction. We collapsed contrast values across the left and right amygdala because threat vs. safe early and threat vs. safe late activation was correlated across the left and right amygdala, $r$ 's $>.28, p$ 's $<.05$. In the presence of the partner, compared to a friend there was significantly reduced threat vs safe activation in the amygdala, $p=.013$, during late trials. Furthermore, in the presence of a partner versus a friend, threat vs safe activation in the amygdala significantly dropped across early to late trials, $p=.006$.

\section{Discussion}

We show that the neural mechanisms underlying threat regulation are different depending on the presence of a romantic partner versus friend. During anticipation of shock, in the presence of a partner (versus friend), there was a decrease in threat-related activity over time in the amygdala. Subgenual anterior cingulate cortex and ventromedial prefrontal cortex activation, by contrast, was greater in the presence of a romantic partner than a friend for threat versus safe 
Running Title: Social domains

trials, without an effect of time. Participants and their partner affiliates had higher mean scores on self-report of mutual comfort when distressed and confiding, than participants and their friend affiliates, consistent with greater attachment function of partner relationships compared to friendships. Furthermore all participants and affiliates provided higher mean scores on items that asked how much they would show distress and look for comfort from partners compared to friends, indicating shared beliefs about the functions of relationships in the partner and friendship domains.

There were two crucial features of the design of the study that increase the likelihood that the activations observed provided an indication of a domains based organisation of neural activation of threat regulation. First, the differences were elicited in the presence of, rather than physical contact with, the affiliate. We hypothesise that this is likely to reflect an anticipatory regulation, while physical contact reflects 'load sharing'. Second the sample was restricted to those who had both partners and friends of at least three years standing, and the identity of the affiliate was established by random allocation. Thus participants were likely to be making the domains based distinction in their everyday lives, and the results were not confounded by selection effects that might have occurred if participants had been able to chose who accompanied them.

The findings are likely therefore to be comparable to studies that have examined effects of viewing the faces of an affiliate such as that of Eisenberger et al (2011) who found viewing romantic partner pictures while receiving painful stimulation led to increased activity in the ventromedial prefrontal cortex. By contrast studies of hand holding such as Coan et al. (2006; 2017), using a threat of shock 
Running Title: Social domains

experiment, finding reduced activation in inferior and lateral prefrontal areas are likely to reflect joint "load sharing" regulatory activity.

In the context of threat of shock, the amygdala has been suggested to signal threat (and more broadly salience), and the parts of the medial prefrontal cortex (including the subgenual anterior cingulate cortex and ventromedial prefrontal cortex) has been suggested to signal safety. In the presence of a romantic partner, in spite of continuing exposure to threat cues, activity in the amygdala fell, while activity in the subgenual anterior cingulate cortex and ventromedial prefrontal cortex remained constant. In the presence of a friend by contrast, continued exposure to threat cues did not lessen amygdala activation which was substantially higher during the late trials compared to the presence of a partner. Thus, in the presence of a partner compared to a friend, individuals may implement a regulatory strategy that is more suited to sustained threat.

We searched for activation within the subgenual anterior cingulate cortex and ventromedial prefrontal cortex, as both of these areas have been implicated in threat regulation, particularly safety-signalling (Etkin et al., 2011). Given the presence of partners and friends in the current experiment, we were examining threat regulation in a social context. Therefore, our findings of increased subgenual cingulate cortex for participants in the presence of partners versus friends whilst receiving threatening stimuli, may have wider implications than safety-signalling. Notably, the subgenual anterior cingulate cortex has also been shown to be involved in a range of social processes including altruistic decision-making (FeldmanHall, Dalgleish, Evans, \& Mobbs, 2015), and group belongingness (Bortolini et al., 2017; Rüsch et al., 2014), and in modulation of affiliative behavior, in part through the effects of oxytocin and 
Running Title: Social domains

arginine vasopressin (McCall \& Singer, 2012). This suggests a tight fit between emotion regulatory and affiliative processes in the demarcation of partner and friends domains. The subgenual cingulate cortex is also one of the key brain regions involved in the pathophysiology of major depressive disorder (Green, Ralph, Moll, Deakin, \& Zahn, 2012; Johansen-Berg et al., 2007; Pulcu et al., 2014). Furthermore, reduced subgenual volumes are associated with chronic social adversity which is associated with increased vulnerability to depression (Ansell, Rando, Tuit, Guarnaccia, \& Sinha, 2012), and depressed adolescents show increased subgenual activation to rejection compared to controls (Silk et al., 2013) implicating the subgenual region in the interplay between social processes and depression.

Strengths of the study included the sampling method that was discussed earlier which made it likely that the groups assessed with partner and friends were comparable, and the comparison of early and late activations to threat of shock to examine the dynamics of threat regulation by social domain. For examination of the key question of the different contributions of romantic partners and friends to threat regulation the study was limited by a between-groups, rather than within individuals, comparison. Thus even though we took the steps outlined earlier to create comparable groups, they may have differed in ways that were not reflected in our measures, a problem that would not arise in a within individuals design. Confounding of type of relationship with gender of affiliate was an unavoidable problem. As we found in this study, if one asks a woman to nominate a friend she is likely to nominate another woman. This would not have been solved by asking her to nominate a male friend because, as substantial evidence shows (e.g. (BleskeRechek et al., 2012), cross-gender friendships commonly entail romantic wishes and 
Running Title: Social domains

processes, and hence have elements that resemble romantic relationships. As we recruited only females for $\mathrm{MRI}$ participation, in line with previous MRI studies (Coan et al., 2006: Eisenberger et al., 2011), future work is needed to assess the extent to which neural activity differs as a function of social domain in male participants as well.

This study provides a novel contribution to understanding how neural responding to threat varies as a function of who people are with. The findings are consistent with the social domains hypothesis that effective functioning entails the ability to identify accurately the scope of threat regulation in different kinds of relationships. Failure to modulate threat (and more broadly emotion) regulation by social domain may lead to a poor match between attempted regulatory strategies and the type of relationship. The social demarcation between romantic partners and friends identified in this study requires further study in typical individuals. A key next step is to compare activations across relationship domains within the same individuals. Also, as was noted earlier, there have been variations in the pain stimuli, the frequency of pairing of CS to pain, duration of trials, and examination of neural activity across time, all of which may be crucial to understanding the extent of threat to which romantic partner and friendship relationships are adapted. Variations in kinds of affect elicitor also need investigation.

Emotion regulation may be either similar or different depending on relationship type. Clearly, romantic and friend relationships differ in multiple ways, such as how much time is spent with the person, and what aspects of lives are shared, and it remains to be established whether the differences reported here depend on such features, or on the identity of the relationship per se. In this study 
Running Title: Social domains

we ensured that we investigated how individuals make the demarcation between partners and friends by requiring them to have both available. Whether those without partners use emotion regulatory strategies with friends that more resemble those seen with partners remains to be investigated.

The findings are relevant not only to healthy adults but also to processes over development and in relation to psychopathology. Enduring romantic relationships typically become established in late adolescence or early adult life, and so separate study of the threat regulatory functions of other family and peer relationships through childhood and adolescence is needed. As we have shown previously behaviors indicating difficulties in maintaining the partner-friend demarcation are characteristic of borderline personality disorder (Hill et al., 2008; Morse et al., 2009). Studies of the kind reported here offer the potential to identify whether failures to modulate threat regulation strategies by social domain contribute to the affective instability and relationship difficulties seen in this disorder. They can increase understanding of the interface between individual differences in domains based neural organisation and different kinds of social support and adversity in disorders such as depression that are known to entail an interplay between biological and social processes. 
Running Title: Social domains

\section{Acknowledgements}

The authors wish to thank the participants for taking part. This research was funded by the University of Reading. 
Running Title: Social domains

\section{References}

Ansell, E.B., Rando, K., Tuit, K., Guarnaccia, J., \& Sinha, R. (2012). Cumulative adversity and smaller gray matter volume in medial prefrontal, anterior cingulate, and insula regions. Biological Psychiatry, 72(1), 57-64.

Bleske-Rechek, A., Somers, E., Micke, C., Erickson, L., Matteson, L., Stocco, C., et al. (2012). Benefit or burden? Attraction in cross-sex friendship. Journal of Social and Personal Relationships, 29(5), 569-596.

Bolton, D.a.H., J. . (2004). Mind, Meaning and Mental Disorder: The Nature of Causal Explanation in Psychology and Psychiatry 2nd edition. Oxford: Oxford University Press.

Bortolini, T., Bado, P., Hoefle, S., Engel, A., Zahn, R., de Oliveira Souza, R., et al. (2017). Neural bases of ingroup altruistic motivation in soccer fans. Scientific Reports, 7(1), 16122.

Büchel, C., Morris, J., Dolan, R.J., \& Friston, K.J. (1998). Brain systems mediating aversive conditioning: an event-related fMRI study. Neuron, 20(5), 947-957.

Coan, J.A., Beckes, L., Gonzalez, M.Z., Maresh, E.L., Brown, C.L., \& Hasselmo, K. (2017). Relationship status and perceived support in the social regulation of neural responses to threat. Social Cognitive and Affective Neuroscience, 12(10), 1574-1583.

Coan, J.A., Kasle, S., Jackson, A., Schaefer, H.S., \& Davidson, R.J. (2013). Mutuality and the social regulation of neural threat responding. Attachment \& Human Development, 15(3), 303-315. 
Running Title: Social domains

Coan, J.A., Schaefer, H.S., \& Davidson, R.J. (2006). Lending a hand social regulation of the neural response to threat. Psychological Science, 17(12), 1032-1039.

Eisenberger, N.I., Master, S.L., Inagaki, T.K., Taylor, S.E., Shirinyan, D., Lieberman, M.D., et al. (2011). Attachment figures activate a safety signal-related neural region and reduce pain experience. Proceedings of the National Academy of Sciences, 108(28), 11721-11726.

Etkin, A., Egner, T., \& Kalisch, R. (2011). Emotional processing in anterior cingulate and medial prefrontal cortex. Trends in Cognitive Sciences, 15(2), 85-93.

FeldmanHall, O., Dalgleish, T., Evans, D., \& Mobbs, D. (2015). Empathic concern drives costly altruism. Neuroimage, 105, 347-356.

Fischer, H., Wright, C.I., Whalen, P.J., Mclnerney, S.C., Shin, L.M., \& Rauch, S.L. (2003). Brain habituation during repeated exposure to fearful and neutral faces: a functional MRI study. Brain Research Bulletin, 59(5), 387-392.

Green, S., Ralph, M.A.L., Moll, J., Deakin, J.F., \& Zahn, R. (2012). Guilt-selective functional disconnection of anterior temporal and subgenual cortices in major depressive disorder. Archives of General Psychiatry, 69(10), 1014-1021.

Hill, J., Fonagy, P., Safier, E., \& Sargent, J. (2003). The ecology of attachment in the family. Family Process, 42(2), 205-221.

Hill, J., Pilkonis, P.A., \& Bear, I. (Eds.). (2010). Social domains, personality, and interpersonal functioning.: John Wiley \& Sons.

Hill, J., Pilkonis, P.A., Morse, J., Feske, U., Reynolds, S., Hope, H., et al. (2008). Social domain dysfunction and disorganization in borderline personality disorder. Psychological Medicine, 38(01), 135-146. 
Running Title: Social domains

Hill, J., Stepp, S.D., Wan, M.W., Hope, H., Morse, J.Q., Steele, M., et al. (2011).

Attachment, borderline personality, and romantic relationship dysfunction. Journal of Personality Disorders, 25(6), 789-805.

Hill, J., Wren, B., Alderton, J., Burck, C., Kennedy, E., Senior, R., et al. (2014). The application of a domains-based analysis to family processes: implications for assessment and therapy. Journal of Family Therapy, 36(1), 62-80.

Jenkinson, M., Bannister, P., Brady, M., \& Smith, S. (2002). Improved optimization for the robust and accurate linear registration and motion correction of brain images. Neuroimage, 17(2), 825-841.

Johansen-Berg, H., Gutman, D., Behrens, T., Matthews, P., Rushworth, M., Katz, E., et al. (2007). Anatomical connectivity of the subgenual cingulate region targeted with deep brain stimulation for treatment-resistant depression. Cerebral Cortex, 18(6), 1374-1383.

Jones, S. (2008). Demarcation of Emotional Expression across Social Domains: the Role of Attachment and Neuroticism. . (MSc), University of Manchester, Manchester.

Kawamichi, H., Kitada, R., Yoshihara, K., Takahashi, H.K., \& Sadato, N. (2015). Interpersonal touch suppresses visual processing of aversive stimuli. Frontiers in Human Neuroscience, 9.

Kennedy, M., Kreppner, J., Knights, N., Kumsta, R., Maughan, B., Golm, D., et al. (2017). Adult disinhibited social engagement in adoptees exposed to extreme institutional deprivation: examination of its clinical status and functional impact. The British Journal of Psychiatry, 211(5), 289-295. 
Running Title: Social domains

LaBar, K.S., Gatenby, J.C., Gore, J.C., LeDoux, J.E., \& Phelps, E.A. (1998). Human amygdala activation during conditioned fear acquisition and extinction: a mixed-trial fMRI study. Neuron, 20, 937-945.

Maresh, E.L., Beckes, L., \& Coan, J.A. (2013). The social regulation of threat-related attentional disengagement in highly anxious individuals. Frontiers in Human Neuroscience, 7, 515.

McCall, C., \& Singer, T. (2012). The animal and human neuroendocrinology of social cognition, motivation and behavior. Nature Neuroscience, 15(5), 681.

Mischel, W., \& Shoda, Y. (1995). A cognitive-affective system theory of personality: reconceptualizing situations, dispositions, dynamics, and invariance in personality structure. Psychological Review, 102(2), 246.

Morse, J.Q., Hill, J., Pilkonis, P.A., Yaggi, K., Broyden, N., Stepp, S., et al. (2009). Anger, preoccupied attachment, and domain disorganization in borderline personality disorder. Journal of Personality Disorders, 23(3), 240-257.

Pulcu, E., Zahn, R., Moll, J., Trotter, P.D., Thomas, E.J., Juhasz, G., et al. (2014). Enhanced subgenual cingulate response to altruistic decisions in remitted major depressive disorder. Neurolmage: Clinical, 4, 701-710.

Rüsch, N., Bado, P., Zahn, R., Bramati, I.E., de Oliveira-Souza, R., \& Moll, J. (2014). You and your kin: Neural signatures of family-based group perception in the subgenual cortex. Social Neuroscience, 9(4), 326-331.

Sehlmeyer, C., Schöning, S., Zwitserlood, P., Pfleiderer, B., Kircher, T., Arolt, V., et al. (2009). Human fear conditioning and extinction in neuroimaging: a systematic review. PloS one, 4(6), e5865. 
Running Title: Social domains

Silk, J.S., Siegle, G.J., Lee, K.H., Nelson, E.E., Stroud, L.R., \& Dahl, R.E. (2013). Increased neural response to peer rejection associated with adolescent depression and pubertal development. Social Cognitive and Affective Neuroscience, 9(11), 1798-1807.

Smith, S.M. (2002). Fast robust automated brain extraction. Human Brain Mapping, 17(3), 143-155.

Spielberger, C.D., Gorsuch, R.L., Lushene, R., Vagg, P., \& Jacobs, G. (1983). Consulting Psychologists Press, Inc. 2». Palo Alto (CA).

Sroufe, L.A. (1977). Wariness of strangers and the study of infant development. Child Development, 731-746.

Younger, J., Aron, A., Parke, S., Chatterjee, N., \& Mackey, S. (2010). Viewing pictures of a romantic partner reduces experimental pain: Involvement of neural reward systems. PloS one, 5(10), e13309. 
Running Title: Social domains

\section{Figure Captions}

Figure 1: Image depicting threat of shock task design. Examples of threat (top row) and safe (bottom row) trial types. Participants were instructed on threat and safe contingencies before the start of the task.

Figure 2: Significant clusters from the threat of shock task by group (A). In the presence of a romantic partner versus a friend, there was increased ventromedial prefrontal cortex and subgenual cingulate cortex activation to threat vs. safe cues overall (B). The clusters presented are from the contrasts: Partner > Friend (Threat > Safe). Coordinates in MNI space; R, right.

Figure 3: Significant clusters from the threat of shock task by group (A). In the presence of a romantic partner versus a friend, there was a reduction in amygdala activation over time to threat versus safe cues (B). The clusters presented are from the contrasts: Partner $>$ Friend $(\text { Threat }>\text { Safe })_{\text {EARLY }}>($ Threat $>$ Safe $)$ LATE. Coordinates in MNI space; $R$, right. 
Running Title: Social domains

Table 1.

Summary of means (SD) for ratings of attachment and demarcation

\begin{tabular}{lcc}
\hline \hline & Attachment ratings \\
\hline Measure & Partners & Friends \\
\hline Comfort & $9.25(1.33)$ & $6.92(2.49)$ \\
Confiding & $9.54(.81)$ & $7.12(2.57)$ \\
\hline \multicolumn{3}{c}{ Demarcation ratings of comfort-seeking from } \\
& partners and friends \\
\hline Measure & Partners & Friends \\
\hline \multicolumn{3}{c}{ Comfort from Partner } \\
Comfort from Friend & $2.99(1.08)$ & $3.5(.84)$ \\
& $2.09(.96)$ & $2.72(.87)$
\end{tabular}

Note: Partners = participants and their affiliate partners $(n=44)$;

Friends $=$ participants and their affiliate friends $(n=40)$.

Table 2.

Summary of means (SD) for ratings for the threat of shock task

\begin{tabular}{|c|c|c|c|c|}
\hline \multirow[b]{3}{*}{ Measure } & \multicolumn{4}{|c|}{ "Threat of Shock } \\
\hline & \multicolumn{2}{|c|}{ Partner } & \multicolumn{2}{|c|}{ Friend } \\
\hline & $\begin{array}{c}\text { Thre } \\
\text { at }\end{array}$ & Safe & $\begin{array}{c}\text { Thre } \\
\text { at }\end{array}$ & Safe \\
\hline Valence Rating & $\begin{array}{c}5.73 \\
(1.61 \\
)\end{array}$ & $\begin{array}{c}6.68 \\
(1.7 \\
3)\end{array}$ & $\begin{array}{c}4.73 \\
(1.82 \\
)\end{array}$ & $\begin{array}{c}6.84 \\
(1.9 \\
5)\end{array}$ \\
\hline Arousal Rating & $\begin{array}{c}4.77 \\
(2.18 \\
)\end{array}$ & $\begin{array}{c}2.91 \\
(2.5 \\
4)\end{array}$ & $\begin{array}{c}5.79 \\
(1.90 \\
)\end{array}$ & $\begin{array}{c}2.73 \\
(2.1 \\
6)\end{array}$ \\
\hline
\end{tabular}




\section{Table 3}

Regional activation patterns in response to stimuli presented in the threat of shock task

\begin{tabular}{|c|c|c|c|c|c|c|c|}
\hline \multirow[t]{2}{*}{ Contrast } & \multirow[t]{2}{*}{ Brain region } & \multirow[t]{2}{*}{$\mathrm{BA}$} & \multirow{2}{*}{$\begin{array}{l}\text { Voxels } \\
\left(\mathrm{mm}^{3}\right)\end{array}$} & \multirow[t]{2}{*}{$\begin{array}{l}\text { Max } \\
\text { Z }\end{array}$} & \multicolumn{3}{|c|}{ Location of $\max Z$} \\
\hline & & & & & $x$ & y & z \\
\hline (Threat > Safe) & $\begin{array}{l}\text { L Insula, L Frontal Operculum Cortex, L Frontal } \\
\text { Orbital Cortex }\end{array}$ & $47 / 44$ & 369 & 5.8 & -44 & 16 & 0 \\
\hline (Threat > Safe) & $\begin{array}{l}\text { R Insula, R Frontal Operculum Cortex, R Frontal } \\
\text { Orbital Cortex } \\
\text { Precuneous Cortex, Cuneal Cortex, } \\
\text { Supracalcarine Cortex, Lateral Occitpial Cortex, } \\
\text { Occipital Fusiform Gyrus, L Hippocampus R }\end{array}$ & $47 / 44$ & 454 & 7.05 & 36 & 22 & 6 \\
\hline (Safe > Threat) & Hippocampus & $7,18,19$ & 23828 & 6.28 & 10 & -58 & 4 \\
\hline Partner > Friend (Threat > Safe) & Apriori: Subgenual Anterior Cingulate Cortex & & 10 & 2.97 & 0 & 20 & -10 \\
\hline Partner > Friend (Threat > Safe) & Apriori: Ventromedial prefrontal cortex & & 9 & 3.74 & -6 & 52 & -16 \\
\hline $\begin{array}{l}\text { Partner }>\text { Friend }(\text { Threat }>\text { Safe })_{\text {EARLY }}>(\text { Threat }> \\
\text { Safe) LATE }\end{array}$ & Apriori: L Amygdala & & 45 & 3.39 & -28 & -8 & -16 \\
\hline $\begin{array}{l}\text { Partner }>\text { Friend }(\text { Threat }>\text { Safe })_{\text {EARLY }}>(\text { Threat }> \\
\text { Safe) })_{\text {LATE }}\end{array}$ & Apriori: R Amygdala & & 5 & 3.51 & 24 & 2 & -22 \\
\hline
\end{tabular}

Note: Corrected cluster for multiple comparisons at $p<0.05$. BA = Brodmann Area. Location of cluster's maximum $\mathrm{Z}$ are in $\mathrm{MNI}$ space. $\mathrm{R}=$ right; $\mathrm{L}=$ left. 


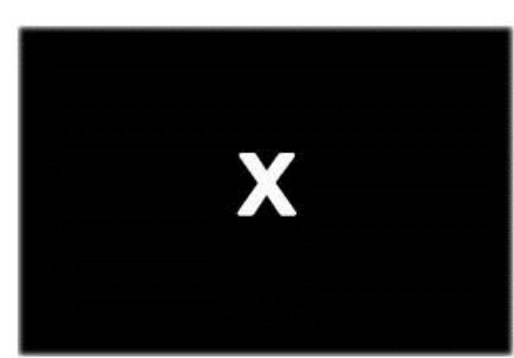

Threat Cue

$1 \mathrm{sec}$

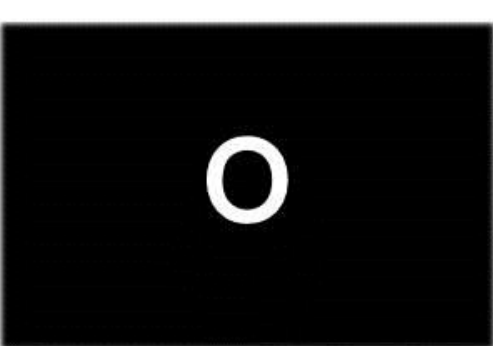

Safe Cue

$1 \mathrm{sec}$

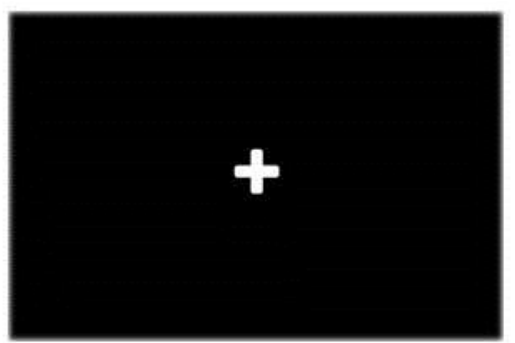

Anticipation

$8 \mathrm{sec}$

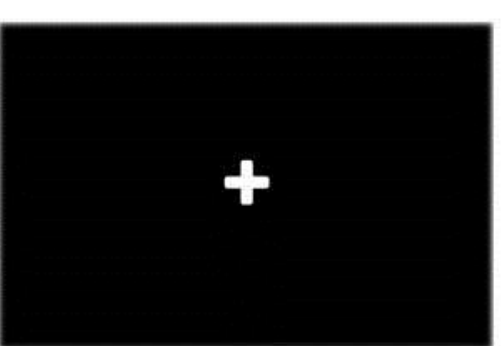

Anticipation

$8 \mathrm{sec}$

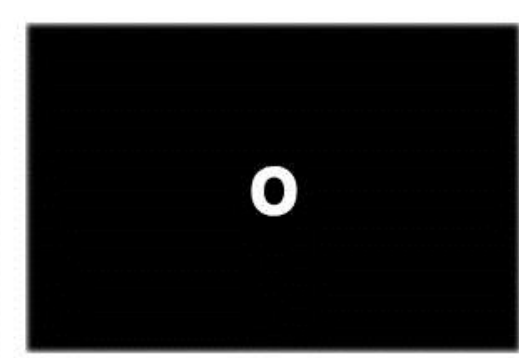

End Cue

$1 \mathrm{sec}$

(50\% shock

probability)

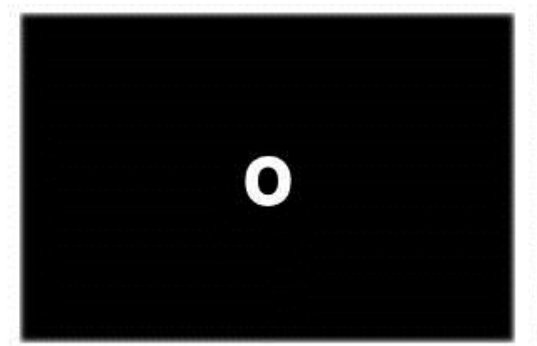

End Cue

No shock

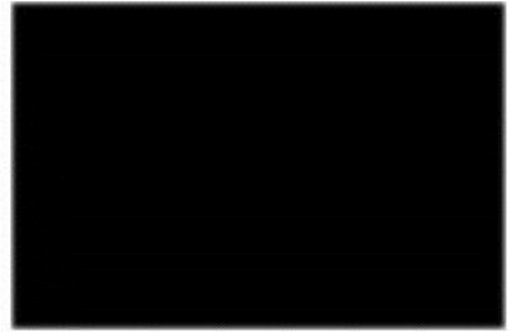

Rest

4-6 sec

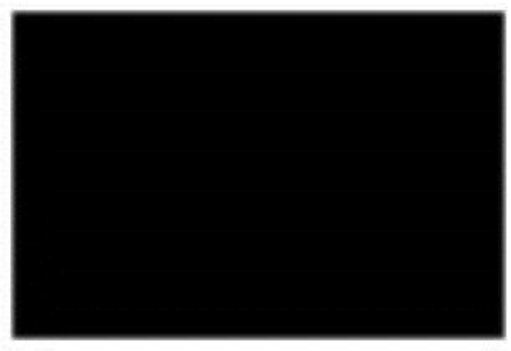

Rest

4-6 sec

Figure 1 
Running Title: Social domains

A
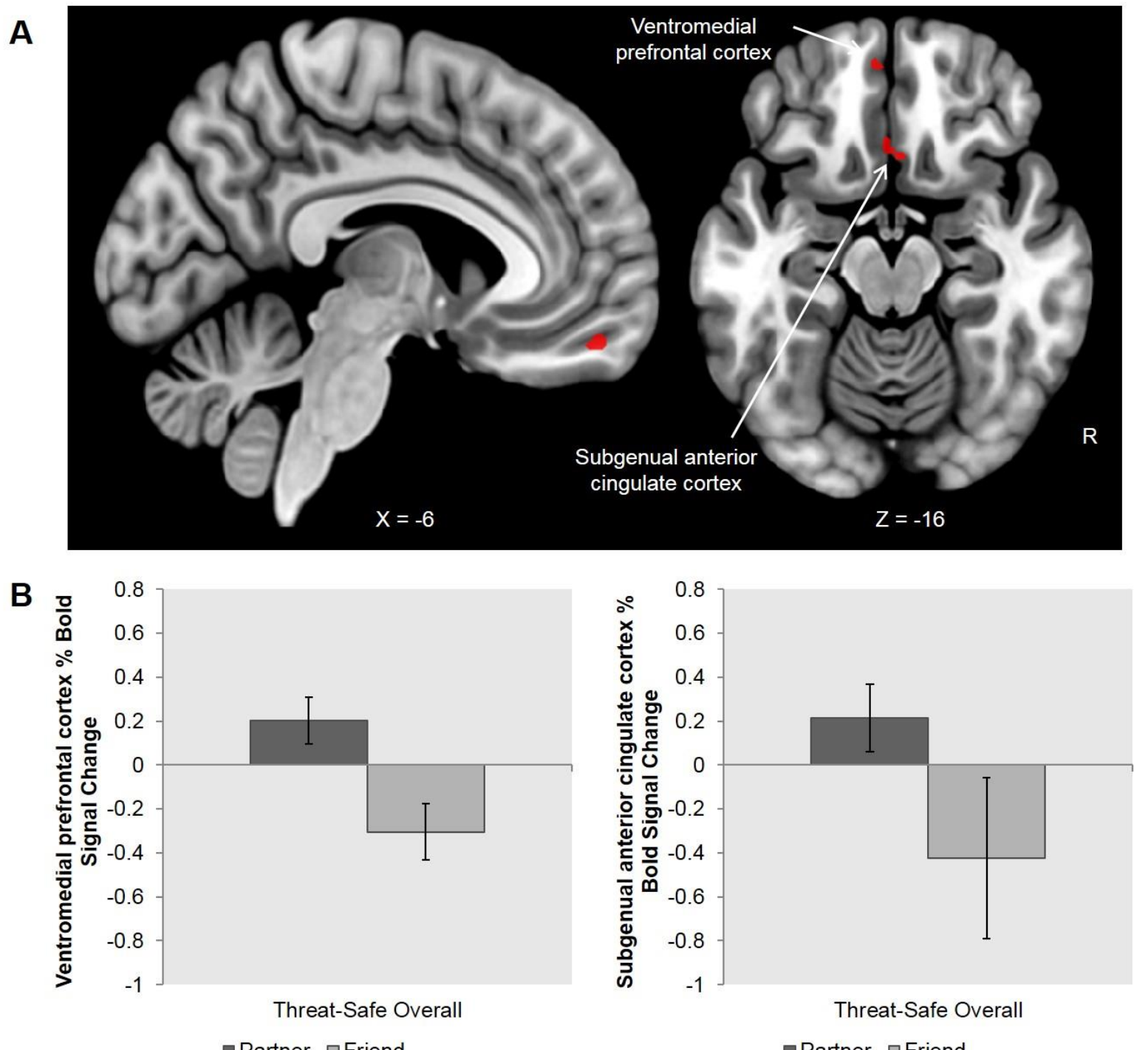

Figure 2

- Partner aFriend 
Running Title: Social domains
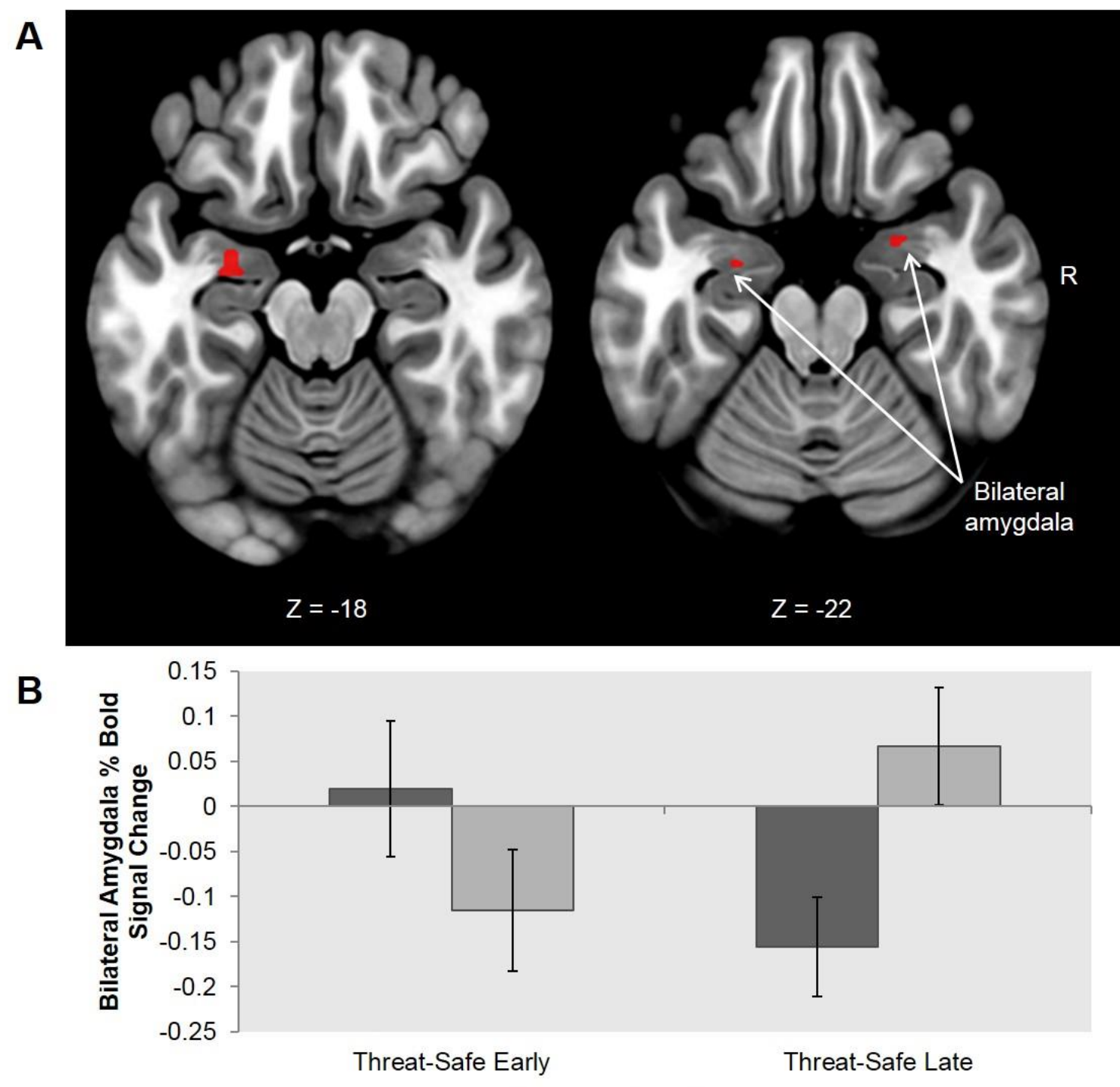

Figure 3

a Partner $\square$ Friend 